the expected persistent current. The velocities of third sound relative to the substrate in the two directions would have been different in the presence of a persistent flow, but no such phenomenon was observed during a series of very careful experiments. In fact, the authors concluded that any such flow, if it existed, was occurring at velocity of less than $1 \mathrm{~cm} \mathrm{~s}^{-1}$.

In the Amherst experiment both the geometry and the method of initiating the film superflow were entirely different. Rather than on the outside of a cylinder, the film was made to flow along the inside of a $37 \mathrm{~cm}$ length of $1.37 \mathrm{~mm}$ diameter stainless steel tube, closed on itself so as to form a continuous loop. Also connected to the loop, by separate short copper tubes, were the annular spaces in two pairs of coaxial cylinder capacitors, $c_{1}$ and $c_{2}$. On the $1.2 \mathrm{~cm}$ length of the loop between these two connections was wound a heater which, when energised, evaporated the film and prevented it flowing past that point, thus acting as a sort of switch for the superflow. At the beginning of an experiment $c_{1}$ and $c_{2}$ were partly filled with superfluid helium so that the tubes above them and the stainless steel loop were covered with the film. In coming to equilibrium the liquid levels were, of course, equalised by film superflow. By measuring the capacities of $c_{1}$ and $c_{2}$ it was possible to determine to a very high precision the amount of dielectric, and thus the level of the liquid helium inside each of them.

To initiate film flow, the heater was energised and a d.c. voltage was applied for a short while across $c_{2}$. The immediate result was a flow of the film out of $c_{1}$, around almost all of the stainless steel loop (except, of course, the short length between $c_{1}$ and $c_{2}$ which incorporated the heater), and into $c_{2}$. A well known property of film flow between reservoirs is the tendency to overshoot when the levels equalise, resulting in slowly damped oscillations with a period of several seconds. Thus, the disturbance of the levels in $c_{1}$ and $c_{2}$ was followed by an oscillatory flow between them through the connecting helium film. By de-energising the heater at an appropriate moment the loop circuit was completed for superflow and, though the level oscillations gradually decayed away, it was hoped that a persistent current might be left in the loop.

To test for the presence of a persistent current after a lapse of time, the heater was again energised, thus opening the loop circuit, so that any kinetic energy flow in the film would have to go into depressing the level in the capacitor on the upstream side of the heater and therefore exciting oscillations once more. In practice level oscillations did indeed occur, even after a lapse of as long as seven hours; and the amplitude of the oscillations did not vary to a measurable extent with the waiting time. The implication must be that Galkiewicz and Hallock had succeeded for the first time in setting up a persistent state of flow around the loop, quite analagous to the persistent electrical current which can be set up in a closed circuit of superconductor : in each case there is a macroscopic flow of particles which apparently persists indefinitely without any dissipation of energy.

Why, on the other hand, was Wang and Rudnick's carefully designed experiment a failure? The reason is not yet known but, on the basis of the present experiment, it is unlikely to be connected with momentum exchange between the flowing film and the helium vapour with which it is in dynamic equilibrium, as had been suggested earlier: this same situation also pertained in the successful Amherst experiment. It is to be hoped that further developments of Galkiewicz and Hallock's new technique will yield the answer to this and other problems connected with film superfluidity.

\section{Changes in enzyme activity during plant photomorphogenesis}

\section{from our Plant Cell Physiology}

Correspondent

Profound changes in the activity of many plant enzymes occur during photomorphogenesis, the response of dark-grown plants to light. Most changes are brought about by red or far-red light acting through the phytochome sytem; in some instances a photoreceptor absorbing blue light is involved. Typically the activity of enzymes already present in dark-grown plant tissue is stimulated above the basal level within hours. Although other phytochrome-controlled responses occur more rapidly, this system is very convenient for studying the regulation of enzyme activity during plant development. Using the density-labelling technique several groups have obtained evidence suggesting that at least two mechanisms are involved-de novo enzyme synthesis and the activation of pre-existing inactive enzymes.

The essence of the density-labelling technique is simple. In order to test whether an increase in enzyme activity in response to a given stimulus is due to synthesis, a heavy isotope such as ${ }^{18} \mathrm{O},{ }^{2} \mathrm{H}$ or ${ }^{15} \mathrm{~N}$ is briefly supplied to the tissue at the same time as the stimulus. A protein extract is later prepared from the tissue and fractionated by buoyant density in $\mathrm{CsCl}$ using an ultracentrifuge. If the degree of labelling of the enzyme being studied is significantly greater in the stimulated tissue than in control tissue this indicates an enhanced rate of de novo synthesis.

Attridge, Johnson and Smith (Biochim. Biophys. Acta, 343, 440; 1974) have studied the phytochrome-controlled increase in activity of phenylalanine ammonia-lyase (PAL) in mustard cotyledons. They have shown, using ${ }^{2} \mathrm{H}_{2} \mathrm{O}$, that PAL and acid phosphatase become density labelled in cotyledons of both irradiated and dark-grown plants, indicating that enzyme synthesis is taking place in all cases. The degree of density labelling of acid phosphatase is unaffected by irradiation, whereas if PAL labelling is measured during the time that the enzyme activity increases in response to light the density labelling of the enzyme is actually lower than in dark grown plants. The authors suggest that the increase in enzyme activity detected in the light is due to the activation of previously synthesised enzyme, although a decrease in the rate of degradation of the enzyme could also be involved. Similar results were obtained for the blue-light controlled increase in PAL activity in gherkin hypocotyls (Attridge and Smith, Biochim. Biophys. Acta, 343, 452; 1974) and the authors cite evidence for the presence of a substance in this tissue which reversibly inactivates PAL.

Another enzyme that increases in activity in irradiated mustard cotyledons is ascorbic acid oxidase. Attridge (Biochim. Biophys. Acta, 362, 258; 1974) has shown that when dark-grown plants are transferred to ${ }^{2} \mathrm{H}_{2} \mathrm{O}$ and irradiated with far-red light, the density of the enzyme increases to the maximum observed within six hours (much more rapidly than the dark control). This evidence strongly suggests that ascorbic acid oxidase, unlike PAL, is synthesised more rapidly in response to light. Increased ribonuclease activity in lupin hypocotyls also seems to be due to phytochrome-controlled stimulation of enzyme synthesis according to Acton and Schopfer (Biochem. J., 142, 449; 1974). These workers were able to demonstrate a greater increase in density of the enzyme in illuminated plants compared to the dark-grown controls. Band-broadening of the enzyme peak in $\mathrm{CsCl}$ also provided evidence for the presence of a population of differently deuterated enzyme molecules-to be expected if the density marker takes a significant time to equilibrate with the amino acid pool. The regulatory mechanisms governing the synthesis of these enzymes remain to be determined. 